\title{
Genetic Risks and Genetic Model Specification
}

\author{
Gang Zheng, ${ }^{1 *}$, Wei Zhang ${ }^{2 *}$, Jinfeng $\mathrm{Xu}^{3}$, Ao $\mathrm{Yuan}^{4, \dagger}$, Qizhai $\mathbf{L i}^{2}$ and \\ Joseph L. Gastwirth ${ }^{5}$ \\ ${ }^{1}$ National Heart, Lung and Blood Institute, Bethesda, MD, USA; \\ ${ }^{2}$ Key Laboratory of Systems and Control, Academy of Mathematics and Systems \\ Science, Chinese Academy of Sciences, Beijing, China; \\ ${ }^{3}$ The University of Hong Kong, Hongkong; \\ ${ }^{4}$ Georgetown University, Washington DC, USA; \\ ${ }^{5}$ George Washington University, Washington DC, USA.
}

\begin{abstract}
Genetic risks and genetic models are often used in design and analysis of genetic epidemiology studies. A genetic model is defined in terms of two genetic risk measures: genotype relative risk and odds ratio. The impacts of choosing a risk measure on the resulting genetic models are studied in the power to detect association and deviation from Hardy-Weinberg equilibrium in cases using genetic relative risk. Extensive simulations demonstrate that the power of a study to detect associations using odds ratio is lower than that using relative risk with the same value when other parameters are fixed. When the HardyWeinberg equilibrium holds in the general population, the genetic model can be inferred by the deviation from Hardy-Weinberg equilibrium in only cases. Furthermore, it is more efficient than that based on the deviation from HardyWeinberg equilibrium in all cases and controls.
\end{abstract}

Key words: Case-control design, Genetic model, Genetic risk, Hardy-Weinberg equilibrium.

*Co-first authors. †For correspondence: Ao Yuan, Georgetown University, Washington DC, USA. E-mail:yuanao@hotmail.com 


\section{Introduction}

In the last several decades, genome-wide association studies (GWAS) have been considered as a big success in searching for the deleterious genetic susceptibilities and hundreds of complex traits have been reported to be associated with the genetic variants, such as obesity (Locke et al., 2015), type 1 diabetes (Todd et al. 2007), type 2 diabetes (Altshuler et al., 2000), carcinoid heart disease (CHD) (Korse et al., 2009). Association studies are a major tool for identifying genes conferring susceptibility to complex traits. These traits are termed complex because they are influenced by both genetic and environmental factors. In the design and analysis of genetic association studies, genetic models are often used. A genetic model refers to Mendel's mode of inheritance (Vogel and Motulsky, 1986). Mendel observed 50\% heterozygote $A B$ and $25 \%$ homozygote of each parental type $A A$ or $B B$ from the crossing of two heterozygotes $A B \times A B$. He found that the phenotype is not always determined by a genotype, and called the allele that determines the phenotype of $A B$ dominant, other allele recessive.

A genetic model is a functional relationship of a risk measure given genotypes. For a binary trait, e.g. case-control data, the risk measure is genotype relative risk (GRR) or odds ratio (OR). It should be noted that for a case-control study, the relative risk can be only approximately estimated for a rare disease under the assumption of Hardy-Weinberg Equilibrium. For a quantitative trait, the risk measure is based on the means of the trait given genotypes. In testing a genetic association, specifying a genetic model is equivalent to specifying an alternative hypothesis. Design and analysis with a correctly specified alternative hypothesis are generally more powerful than those with a more broad alternative hypothesis. On the other hand, if the model is incorrectly specified, the outcomes are not satisfactory. Thus, how to specify a correct genetic model and avoid specifying a wrong genetic model are important in both design and analysis of genetic association studies. In genetics literatures, the genetic models are generally classified in term of two measures: genotype relative risk and odds ratio. However, no existing literatures have compared the influence of the two risk measures in the genetic association studies. 
The Hardy-Weinberg equilibrium is a very important law in the analysis of genetic data. Assume for a biallelic locus with the allele $A$ and $B$. Denote the genotypes by $\left(G_{0}, G_{1}, G_{2}\right)=(A A, A B, B B)$. The HWE states that under random mating the genotype frequencies in a population satisfy the relations: $\operatorname{Pr}\left(G_{0}\right)=q^{2}, \operatorname{Pr}\left(G_{1}\right)=$ $2 q(1-q), \operatorname{Pr}\left(G_{2}\right)=(1-q)^{2}$, where $q$ is the allele frequency of $A$. If there is no disturbing factors, such as population stratification, genotyping error, the HWE law usually hold in the source population.

In this paper, we examine the roles of using risk measures in specifying genetic models and how they influence the design and analysis of genetic association studies. We focus on case-control studies but the quantitative trait is also briefly discussed. Then we study how to specify a genetic model using the information of derivation from Hardy-Weinberg equilibrium in cases.

\section{Risk Measures and Genetic Models}

\section{$2.1 \quad$ Notation}

Consider a disease locus with alleles $A$ and $B$. Denote the genotypes by $\left(G_{0}, G_{1}, G_{2}\right)=$ $(A A, A B, B B)$, the disease prevalence by $\kappa$, and the penetrance by $f_{i}=\operatorname{Pr}\left(D \mid G_{i}\right)$ $(i=0,1,2)$, where $D$ stands for the disease. In a case-control association study, the counts for $G_{i}$ in $r$ cases and $s$ controls are denoted by $r_{i}$ and $s_{i}$, respectively $(i=0,1,2)$. Denote $n_{i}=r_{i}+s_{i}, i=0,1,2$ and $n=n_{0}+n_{1}+n_{2}$.

For a quantitative trait, let the random trait be $T=\mu+g+\epsilon$, where $\mu$ is the mean in the absence of the genetic effect, $g$ is the genetic effect with $g=-a, d$ and $a$ ( $a$ and $d$ are two constants, $a>0$ and $-a \leq d \leq a$ ) for genotypes $G_{0}, G_{1}$ and $G_{2}$, and $\epsilon$ is a random error with 0 mean. Denote $\mu_{i}=\mathrm{E}\left(T \mid G_{i}\right)$, the conditional mean given the genotype $(i=0,1,2)$. 


\subsection{Binary Traits}

The GRRs and ORs are denoted by $\mathrm{GRR}_{i}=f_{i} / f_{0}$ and $\mathrm{OR}_{i}=\left\{f_{i}\left(1-f_{0}\right)\right\} /\left\{f_{0}\left(1-f_{i}\right)\right\}$ $(i=1,2)$. The null hypothesis is $H_{0}: \mathrm{GRR}_{1}=\mathrm{GRR}_{2}=1$ or $H_{0}: \mathrm{OR}_{1}=\mathrm{OR}_{2}=1$. Genetic models are only relevant under the alternative hypothesis $H_{1}$. Without loss of generality, assume $B$ is the risk allele. Using the GRRs, the genetic model is recessive $(\mathrm{REC})$ if $\mathrm{GRR}_{1}=1$, additive $(\mathrm{ADD})$ if $\mathrm{GRR}_{1}=\left(1+\mathrm{GRR}_{2}\right) / 2$, and dominant $(\mathrm{DOM})$ if $\mathrm{GRR}_{1}=\mathrm{GRR}_{2}$. The definitions of genetic models using ORs are similar.

The alternative hypothesis $H_{1}$ can be specified in terms of a genetic model, including the three common genetic models, using GRRs with $x$ or ORs with $y$ as

$$
\begin{aligned}
& H_{1}(x)=\left\{\left(\mathrm{GRR}_{1}, \mathrm{GRR}_{2}\right) \neq(1,1): \mathrm{GRR}_{1}=1-x+x \mathrm{GRR}_{2}, x \in[0,1]\right\} ; \\
& H_{1}(y)=\left\{\left(\mathrm{OR}_{1}, \mathrm{OR}_{2}\right) \neq(1,1): \mathrm{OR}_{1}=1-y+y \mathrm{OR}_{2}, y \in[0,1]\right\},
\end{aligned}
$$

The REC, ADD and DOM models correspond to $x=0, x=1 / 2$ and $x=1$ in $H_{1}(x)$ and $y=0, y=1 / 2$ and $y=1$ in $H_{1}(y)$, respectively. Specifying $x=x_{0} \in[0,1]$ or $y=y_{0} \in[0,1]$ is equivalent to specify an alternative hypothesis $H_{1}\left(x_{0}\right)$ and $H_{1}\left(y_{0}\right)$. Then sample size/power and analysis can be done for the specified $H_{1}\left(x_{0}\right)$ or $H_{1}\left(y_{0}\right)$ rather than for $H_{1}(x)$ or $H_{1}(y)$ with all $x \in[0,1]$ or $y \in[0,1]$.

The first question to address is that whether or not the genetic models defined using GRRs and ORs are equivalent. The following properties of GRRs and ORs can be readily verified: (i) $\mathrm{GRR}_{2} \geq \mathrm{GRR}_{1}$ if and only if $\mathrm{OR}_{2} \geq \mathrm{OR}_{1}$ and $\mathrm{GRR}_{1} \geq 1$ if and only if $\mathrm{OR}_{1} \geq 1$, where all the equalities hold simultaneously; (ii) $\mathrm{OR}_{2}>\mathrm{GRR}_{2}$ and $\mathrm{OR}_{1} \geq \mathrm{GRR}_{1}$, where the equality holds under the REC model; (iii) if $\mathrm{GRR}_{1}=$ $\left(1+\mathrm{GRR}_{2}\right) / 2$, then $\mathrm{OR}_{1}<\left(1+\mathrm{OR}_{2}\right) / 2$; and (iv) let $x$ and $y$ be given in equation (1) and equation (2), then $y \leq x$, where the equality holds under either REC or DOM models.

Note that (i) implies that the REC or DOM models can be defined in terms of either GRRs or ORs. However, from (iii), the ADD model defined using GRRs differs from that defined using ORs. Further, from (ii), the ORs are always larger than the GRRs given the same genetic model. (iv) further implies, except for the REC 
and DOM models, any other model defined using the GRRs with $x$ corresponds to a different model defined using the ORs with $y$ and that $y<x$. In other words, only the REC and DOM models do not depend on the risk measures. Other models between the REC and DOM models are only well defined given the risk measure.

Table 1 shows the values of $y$ given $x=0,1 / 2,1$, minor allele frequencies (MAFs) and $\kappa$. We assume that the Hardy-Weinberg Equilibrium (HWE) proportion holds in the source population. It indicates that MAF and $\kappa$ slightly affect the ORs given the GRRs, but a larger $\kappa$ would increase the ORs for fixed GRRs. The results in Table 1 have an implication in the design of association studies. For example, under the REC model with MAF $=0.3, \kappa=0.10$, the power to detect GRR $=1.5$ is the same as that to detect $\mathrm{OR}=1.584$ with the same sample size. In other words, given the same sample size, the power of a study to detect $\mathrm{OR}_{2}=1.5$ would be lower than that with $\mathrm{GRR}_{2}=1.5$. For illustration, we assume $\kappa=0.05, r=s=1000$, and a two-sided trend test. The powers to detect $\mathrm{OR}_{2}=1.5$ under the ADD model are $34.9 \%, 68.9 \%, 87.2 \%$, and $89.4 \%$ for $\mathrm{MAF}=0.05,0.15,0.30$, and 0.45 , respectively, while the powers to detect $\mathrm{GRR}_{2}=1.5$ under the same genetic model are $41.9 \%$, $75.9 \%, 90.1 \%$, and 92.5\%, respectively. The power differences using GRRs or ORs could be quite substantial.

Table 1 about here

Both GRRs and ORs are commonly used in the literature for sample size and power calculations (Slager and Schaid, 2001; Freidlin et al., 2002; Jackson et al., 2002; Pfeilffer and Gail, 2003) and for deriving test statistics (Sasieni,1997; Chen and Chaterjee, 2007). Our results indicate that in design of case-control genetic association studies, one should pay attention to the choice of a risk measure in calculating power and sample size, especially the same risk measure should be used when comparing different study designs and analysis. The GRRs and ORs have a one-to-one relationship given $f_{0}$. Given $\mathrm{GRR}_{i}, \mathrm{OR}_{i}=\operatorname{GRR}_{i}\left(1-f_{0}\right) /\left(1-\mathrm{GRR}_{i} f_{0}\right)(i=0,1,2)$. Given $\mathrm{OR}_{i}, \mathrm{GRR}_{i}=\mathrm{OR}_{i} /\left(\mathrm{OR}_{i} f_{0}+1-f_{0}\right)(i=0,1,2)$. With these formulas, one can convert from GRRs to ORs and vice versa. 
We have discussed genetic models and risk measures at a disease locus. In practice, only a marker locus is observed, which is assumed to be in linkage disequilibrium (LD) with the disease locus. The $\mathrm{LD}$ is often measured by $D^{\prime} \in[-1,1]$, which is a standardized LD parameter (Lewontin, 1964). Suppose that the marker locus has two alleles, denoted them by $a$ and $b$, with allele frequency $\operatorname{Pr}(b)=q$. Denote the corresponding genotypes by $\left(G_{0}^{*}, G_{1}^{*}, G_{2}^{*}\right)=(a a, a b, b b)$. As defined above, the disease locus has two alleles $A$ and $B$ and three genotypes $\left(\left(G_{0}, G_{1}, G_{2}\right)=(A A, A B, B B)\right)$. Denote the joint probabilities of the two loci by $\operatorname{Pr}(A a), \operatorname{Pr}(A b), \operatorname{Pr}(B a)$, and $\operatorname{Pr}(B b)$. Let $D=\operatorname{Pr}(A a) \operatorname{Pr}(B b)-\operatorname{Pr}(A b) \operatorname{Pr}(B a)$. Then the measure $D^{\prime}$ can be defined by

$$
D^{\prime}=\left\{\begin{array}{cl}
\frac{D}{\min (p(1-q),(1-p) q)}, & \text { if } D>0 ; \\
\frac{D}{\min (p q,(1-p)(1-q))}, & \text { if } D \leq 0 .
\end{array}\right.
$$

If the marker and disease loci are under complete LD (i.e., $\left|D^{\prime}\right|=1$ with the same allele frequency), the above discussions and results can be applied to the marker locus. However, the LD is often incomplete. The joint probabilities of $\operatorname{Pr}(A a), \operatorname{Pr}(A b), \operatorname{Pr}(B a)$, and $\operatorname{Pr}(B b)$ are given in Table 1 in Zheng et al. (2009) and they only studied genetic models under incomplete LD using GRRs. Using these joint probabilities, we can calculate the conditional probabilities $\operatorname{Pr}\left(G_{j}^{*} \mid G_{i}\right), i, j=0,1,2$. Then based on them, the penetrances for the marker locus $f_{0}^{*}=\operatorname{Pr}\left(\operatorname{case} \mid G_{0}^{*}\right), f_{1}^{*}=\operatorname{Pr}\left(\operatorname{case} \mid G_{1}^{*}\right)$ and $f_{2}^{*}=\operatorname{Pr}\left(\right.$ case $\left.\mid G_{2}^{*}\right)$ can be calculated by $f_{i}^{*}=\sum_{j=0}^{2} f_{j} \operatorname{Pr}\left(G_{j}^{*} \mid G_{i}\right), i=0,1,2$. Thus we can obtain the $\mathrm{GRR}_{1}, \mathrm{GRR}_{2}, \mathrm{OR}_{1}$, and $\mathrm{OR}_{1}$ at the marker locus. For a given disease prevalence and MAF, Table 2 reports ORs and GRRs at the marker locus when the true genetic model is defined using GRRs at the disease locus. ORs are still greater than GRRs under all three models when $D^{\prime}<1$. In addition, the REC/DOM models are no longer the same models at the marker locus. This, in conjunction with the results that we presented before, implies that the choice of a risk measure is even more relevant when a marker locus is used.

Table 2 about here 


\subsection{Quantitative Traits}

In this part, we only consider the disease locus. For a quantitative trait $T$, the REC, $\mathrm{ADD}$ and DOM models are defined as $d=-a, d=0$ and $d=a$, respectively, where $|d| \leq a$. The null hypothesis is given by $H_{0}: a=0$ or $H_{0}: \mu_{0}=\mu_{1}=\mu_{2}=\mu$. Without loss of generality, assume $\mu=0$. The $H_{1}$ can be written in terms of a genetic model as

$$
H_{1}(z)=\left\{\left(\mu_{0}, \mu_{1}, \mu_{2}\right) \neq(0,0,0): \mu_{1}=(1-z) \mu_{0}+z \mu_{2}, z \in[0,1]\right\}
$$

as compared to $H_{1}(x)$ using GRRs and $H_{1}(y)$ using ORs. The REC, ADD and DOM models correspond to $z=0,1 / 2$ and 1 , respectively.

From the above, the conditional mean given the genotype is the risk measure for a quantitative trait in an association study. In this part, we will compare this risk measure with the two risk measures (relative risk and odds ratio) for a binary trait through transforming the quantitative trait into a binary trait with some prespecified thresholds. To link the genetic models for a quantitative trait to those using GRRs and ORs, let a case (control) be such that $T>t_{2}\left(T<t_{1}\right)$, where $t_{2}>t_{1}$ are thresholds for cases and controls. The conditional cumulative distribution function (c.d.f.) for the quantitative trait $T$ given $G_{i}$ is $T \mid G_{i} \sim F_{\mu_{i}}(t)=F\left(t-\mu_{i}\right)$. The c.d.f. for $T$ is $F_{T}(t)=\sum_{i=0}^{2} g_{i} F_{\mu_{i}}(t)=\sum_{i=0}^{2} g_{i} F\left(t-\mu_{i}\right)$, where $g_{i}$ is the population frequency of $G_{i}$. Note that $f_{0}=1-F\left(t_{2}+a\right), f_{1}=1-F\left(t_{2}-d\right)$ and $f_{2}=1-F\left(t_{2}-a\right)$. Then $x=\left(F\left(t_{2}+a\right)-F\left(t_{2}-d\right)\right) /\left(F\left(t_{2}+a\right)-F\left(t_{2}-a\right)\right)$. Since $F^{\prime \prime}\left(t_{2}\right)>0$ when $t_{2}$ is large enough, $F\left(t_{2}-d\right)>(1-z) F\left(t_{2}+a\right)+z F\left(t_{2}-a\right)$. It follows from $F\left(t_{2}+a\right)-F\left(t_{2}-d\right)<z\left(\left(F\left(t_{2}+a\right)-F\left(t_{2}-a\right)\right)\right)$ that $x<z$. It can be shown that $z \geq x \geq y$, where equalities hold only under the REC or DOM models. The result shows that the definitions of the REC or DOM models for a quantitative trait are the same as those for a transformed binary trait. Thus the REC and DOM models are well defined for a quantitative trait or a binary trait. However, for any other models between the REC and DOM models, this equivalence is not established. So it should be noted that they are defined based on a binary (GRRs or ORs) or quantitative trait. Numerical values of $x$ and $y$ given $z$ when $F$ is a normal distribution are reported in 
Figure 1, where the values of $a$ and $t_{2}=t, t_{1}=-t$ are also given. In each plot, the dashed line for $y$ is below the solid line for $x$, and both are below the diagonal line for $z$ (the diagonal lines are not shown in the figure), which indicates $z>x>y$. Note that the solid and dashed lines are closer when $t$ is larger. This provides insight of a known fact that ORs can be used to approximate GRRs for a rare disease.

Figure 1 about here

\section{Genetic Model Detection}

\subsection{Can Genetic Model be inferred by Trend Test?}

The trend test is widely used for genetic association in the case-control design (Armitage, 1955; Cochran, 1954). It can be obtained as a score test from the logistic regression model, where the genetic effect at a marker locus is coded by 0 for $G_{0}, \theta$ for $G_{1}$, and 1 for $G_{2}$, where $\theta \in[0,1]$. The true genetic model is indexed by $x \in[0,1]$ as in $H_{1}(x)$, which is unknown. (We use GRRs for genetic models here.) The trend test can be written as (Sasieni, 1997),

$$
Z(\theta)=\frac{\left(\frac{r s}{n}\right)^{\frac{1}{2}}\left\{\left(\theta \frac{r_{1}}{r}+\frac{r_{2}}{r}\right)-\left(\theta \frac{s_{1}}{s}+\frac{s_{2}}{s}\right)\right\}}{\left\{\left(\theta^{2} \frac{n_{1}}{n}+\frac{n_{2}}{n}\right)-\left(\theta \frac{n_{1}}{n}+\frac{n_{2}}{n}\right)^{2}\right\}^{\frac{1}{2}}},
$$

where $n_{i}=r_{i}+s_{i}(i=0,1,2)$. When the genetic model is known, $Z(0), Z(1 / 2)$ and $Z(1)$ are used for the REC, ADD and DOM models, respectively. In practice, the true $x$ is unknown.

An interesting question is whether or not $\theta$ that maximizes the score test $Z^{2}(\theta)$ over all $\theta \in[0,1]$ is a good estimate of the underlying genetic model $x$. Denote this $\theta$ as $\hat{\theta}$. Then, from Kimeldorf et al. (1992) and Zheng, Joo, Yang (2009), we have

$$
\hat{\theta}=\frac{r_{1} / n_{1}-r_{0} / n_{0}}{r_{2} / n_{2}-r_{0} / n_{0}}
$$

Table 3 reports $\hat{\theta}$ calculated using (4) in a simulation study from 10,000 replicates for a given genetic model. Except for $\mathrm{MAF}=0.1, \hat{\theta}$ is often close to $x$ as $n$ increases. 
If the true genetic model is defined using the ORs indexed by $y$, the limit of $\hat{\theta}$ is not $y$. Thus, $\hat{\theta}$ is not an unbiased estimate of the genetic model regardless of the risk measure used.

On the other hand, from the definition of $H_{1}(x)$ in (1), the true model is indexed by $x=\left(\mathrm{GRR}_{2}-1\right) /\left(\mathrm{GRR}_{1}-1\right)=\left(f_{1}-f_{0}\right) /\left(f_{2}-f_{0}\right) \in[0,1]$. If $\hat{\theta}$ in $(4)$ is an estimate of $x$, then $f_{i}=\operatorname{Pr}\left(D \mid G_{i}\right)$ would be estimated by $\widehat{f}_{i}=r_{i} / n_{i}(i=0,1,2)$. This estimate using the retrospective case-control data is known to be biased. However, when the sample size $n$ is large enough, $r_{i} / n_{i} \rightarrow \phi p_{i} /\left\{\phi p_{i}+(1-\phi) q_{i}\right\}(i=0,1,2)$, where $\phi$ is the proportion of cases in the data. Replacing $r_{i} / n_{i}$ in $\hat{\theta}$ given in (4) by its limit, $\hat{\theta}$ can be written as

$$
\hat{\theta} \approx x \frac{f_{2}(\phi-k)+k(1-\phi)}{f_{1}(\phi-k)+k(1-\phi)} .
$$

This implies that $\hat{\theta}$ derived by the equation (4) is indeed an estimate of $x$ if $\phi=k$ or $f_{1}=f_{2}$ (the DOM model) or $x=0$ (the REC model). The condition $\phi=k$ implies that the proportion of cases in the data is an unbiased estimate of the disease prevalence, which is very unlikely in case-control studies. As we claimed before, when genetic markers are used, the REC and DOM models at the disease locus are no longer REC and DOM models at the marker locus. Thus, the conditions $f_{1}=f_{2}$ or $x=0$ are rarely satisfied in practice.

Table 3 about here

\subsection{Genetic Model Inference using the Deviation from HWE}

It has been shown that deviation from HWE indicates associations (Nielsen, Ehm, Weir, 1999). Song and Elston (2006) combined the trend test $Z(1 / 2)$ and the test based on the deviation from HWE between cases and controls (denote it by $Z_{C C}$ ) to improve testing association, where

$$
Z_{C C}=\frac{\sqrt{r s / n}\left(\widehat{\Delta}_{\text {case }}-\widehat{\Delta}_{\text {control }}\right)}{\left[1-n_{2} / n-n_{1} /(2 n)\right]\left[n_{2} / n+n_{1} /(2 n)\right]},
$$

$\widehat{\Delta}_{\text {case }}=r_{2} / r-\left[r_{2} / r+r_{1} /(2 r)\right]^{2}$ and $\widehat{\Delta}_{\text {control }}=s_{2} / s-\left[s_{2} / s+s_{1} /(2 s)\right]^{2}$. Zheng and $\operatorname{Ng}(2008)$ used $Z_{C C}$ to detect a genetic model $\theta=\theta^{*}$ from $\theta=0,1 / 2$, or 1 and then 
applied the trend test $Z\left(\theta^{*}\right)$ to detect associations. We can also construct the test statistic based on deviation from HWE in cases only, denote it by

$$
Z_{C}=\frac{\sqrt{r} \widehat{\Delta}_{\text {case }}}{\left[1-n_{2} / n-n_{1} /(2 n)\right]\left[n_{2} / n+n_{1} /(2 n)\right]},
$$

$\widehat{\Delta}_{\text {case }}=r_{2} / r-\left[r_{2} / r+r_{1} /(2 r)\right]^{2}$, and use $Z_{C}$ to detect a genetic model. We compare performance of selecting genetic models correctly using $Z_{C C}$ and $Z_{C}$ using the same thresholds -1.645 and 1.645 as used in Zheng and $\mathrm{Ng}$ (2008).

In the following, we conduct a simulation study to compare the performance of $Z_{C C}$ and $Z_{C}$ in the detection of genetic model. Consider four MAF values for the disease locus (denoted as $\mathrm{p}=0.05,0.15,0.30,0.45$ ) and assume that HWE holds in the general population. Denote the disease prevalence by $\kappa=0.05,0.10$. Let GRR2=1.5. Then under a given genetic model, we can derive GRR1. With the values of MAF, $\mathrm{GRR}_{1}$, and $\mathrm{GRR}_{2}$, we can calculate the genotype frequencies in the case and control group as $\operatorname{Pr}\left(G_{0} \mid\right.$ case $)=\frac{(1-p)^{2}}{\kappa}, \operatorname{Pr}\left(G_{1} \mid\right.$ case $)=\frac{2 p(1-p) \eta \mathrm{GRR}_{1}}{\kappa}, \operatorname{Pr}\left(G_{2} \mid\right.$ case $)=\frac{p^{2} \eta \mathrm{GRR}_{2}}{\kappa}$, and $\operatorname{Pr}\left(G_{0} \mid\right.$ control $)=\frac{(1-p)^{2}(1-\eta)}{1-\kappa}, \operatorname{Pr}\left(G_{1} \mid\right.$ control $)=\frac{(1-p)^{2}(1-\eta) \mathrm{GRR}_{1}}{1-\kappa}, \operatorname{Pr}\left(G_{2} \mid\right.$ control $)=$ $\frac{p^{2}(1-\eta) \mathrm{GRR}_{2}}{1-\kappa}$, where

$$
\eta=\operatorname{Pr}\left(\text { case } \mid G_{0}\right)=\frac{\kappa}{(1-p)^{2}+2 p(1-p) \mathrm{GRR}_{1}+p^{2} \mathrm{GRR}_{2}} .
$$

Then we generate 1000 cases and 1000 controls from two multinomial distributions: $\operatorname{Mul}\left(1000, P_{\text {case }}\right)$ and $\operatorname{Mul}\left(1000, P_{\text {control }}\right)$, respectively, where $P_{\text {case }}=\left(\operatorname{Pr}\left(G_{0} \mid\right.\right.$ case $)$, $\operatorname{Pr}\left(G_{1} \mid\right.$ case $), \operatorname{Pr}\left(G_{2} \mid\right.$ case $\left.)\right)$ and $P_{\text {control }}=\left(\operatorname{Pr}\left(G_{0} \mid\right.\right.$ control $), \operatorname{Pr}\left(G_{1} \mid\right.$ control $), \operatorname{Pr}\left(G_{2} \mid\right.$ control $\left.)\right)$ are two three dimensional vectors. Table 4 reports the correct model selection proportions by $Z_{C C}$ and $Z_{C}$ under a given genetic model. Results show that $Z_{C}$ is significantly more effective to detect true genetic models than $Z_{C C}$ under the REC and DOM models. When $\kappa=0.05$, the frequency to detect a true REC model using $Z_{C}$ is $21.62 \%$ for $\mathrm{MAF}=0.05$ but it increases to $94.18 \%$ for $\mathrm{MAF}=0.45$. The corresponding frequencies using $Z_{C}$ are $15.83 \%(\mathrm{MAF}=0.05)$ and $78.25 \%(\mathrm{MAF}=0.45)$. When the true model is dominant and $\kappa=0.10$, the frequencies of selecting a true DOM model rate using $Z_{C C}$ for $\mathrm{MAF}=0.05,0.15,0.30,0.45$ are $14.04 \%, 48.50 \%, 75.98 \%$, and $80.19 \%$, respectively. When $Z_{C}$ is used, they are $25.13 \%, 67.65 \%, 91.19 \%$, and $94.75 \%$ which are correspondingly larger than those using $Z_{C C}$. 
In reality, the genetic model is usually unknown. In the case-control association studies, we can consider using the statistics $Z_{C C}$ and $Z_{C}$ to choose the genetic model first and then use the test statistics corresponding to the selected genetic model to test for the associations. Zheng and $\mathrm{Ng}$ (2008) proposed a two-phase test statistics based on the Cochran Armitage trend test (Armitage, 1955; Cochran, 1954) for the casecontrol association studies. In details, in the first phase, they used the statistic $Z_{C C}$ based on the deviation from HWE between cases and controls to choose the genetic model and then applied the trend test for the selected genetic model to conduct the association studies in the second phase.

Based on the definitions of GRR and OR, there are one to one correspondence between GRR and OR when $f_{0}$ is fixed. The only difference between GRR and OR for one data is that their values are not the same. Although we only report the results how GRR affects genetic model using $Z_{C}$ and $Z_{C C}$, the results are similar for OR.

Table 4 about here

\section{Conclusions}

We examined the definitions of genetic models using both GRRs and ORs for a binary trait, and showed the differences between them. Case-control data obtained under a threshold model from a quantitative trait is also discussed. Our results show that genetic models using GRRs and ORs are different. For the same genetic model, the OR is always larger than the GRR. In terms of power calculations, the power to detect association using GRRs is greater than that using the ORs while other parameters are the same. Therefore, the measure (GRR or OR) has to be specified clearly when calculating power or sample size and interpreting results.

A genetic model refers to specific mode of inheritance. However, in real applications, how the genetic variant affect the risk of a disease is unknown. Using an improper genetic model might result in a loss of power substantially, especially when the true model is recessive (dominant) and a dominant (recessive) model is used. So 
the detection of genetic model in association studies is very important. We showed that the true genetic model cannot be estimated using the trend test for the casecontrol data. However, it can be detected using the deviation from HWE in cases only, which is more effective than using the deviation from HWE between cases and controls when HWE holds in the general population. In addition, we can incorporate this procedure of genetic model detection into any test methods which are constructed under a specific genetic model and obtain an omnibus test statistic to conduct the association studies. This deserves further research. Besides this, we also can further investigate how the statistic $Z_{C}$ influences the power of the genetic association studies and attempt to construct a high-power test statistic based on $Z_{C}$ directly, and whether the results hold for the markers with Hardy-Weinberg disequilibrium.

\section{Acknowledgements}

We would like to thank three anonymous reviewers for their careful reading and thoughtful comments, which greatly improved our manuscript. The authors would like to thank Ryo Yamada for some helpful discussions. Q. Li was supported in part by the National Science Foundation of China, Grant No. 11371353, 61134013) and the Breakthrough Project of Strategic Priority Program of the Chinese Academy of Sciences, Grant No. XDB13040600.

\section{References}

Altshuler D, Hirschhorn JN, Klannemark M, Lindgren CM, Vohl MC, Nemesh J, Lane CR, Schaffner SF, Bolk S, Brewer C, et al. The common PPARg Pro12Ala polymorphism is associated with decreased risk of type 2 diabetes. Nat Genet $26,76-80 ; 2000$.

Armitage P. Tests for linear trends in proportions and frequencies. Biometrics 11, $375-386 ; 1955$. 
Cochran WG. Some methods for strengthening the common chi-square tests. Biometrics 10, 417-451; 1954.

Chen J, Chaterjee N. Exploiting Hardy-Weinberg equilibrium for efficient screening of single SNP associations from case-control studies. Hum Hered 63, 196-204; 2007.

Freidlin B, Zheng G, Li Z, et al. Trend tests for case-control studies of genetic markers: power, sample size and robustness. Hum Hered 53, 146-152; 2002.

Jackson MR, Genin E, Knapp M, et al. Accurate power approximations for $\chi^{2}$-tests in case-control association studies of complex disease genes. Ann Hum Genet 66, 307-321; 2002.

Kimeldorf G, Sampson AR, Wright LR. Min and max scorings for two-sample ordinal data. J Am Statist Assoc 87, 241-247; 1992.

Korse, CM, Taal, BG, De Groot, CA, Bakker, RH, and Bonfrer, JM. ChromograninA and N-terminal pro-brain natriuretic peptide: an excellent pair of biomarkers for diagnostics in patients with neuroendocrine tumor. J Clin Oncol, 27:42934299; 2009.

Lewontin, RC. The interaction of selection and linkage. I. General considerations; heterotic models. Genetics 49, 49-67; 1964.

Locke, AE, Kahali, B, Berndt, SI, Justice, AE, Pers, TH, Day, FR, Powell, C, Vedantam, S, Buchkovich, ML, Yang, J, et al. Genetic studies of body mass index yield new insights for obesity biology. Nature, 518:197-206; 2015.

Nielsen DM, Ehm MG, Weir BS. Detecting marker-disease association by testing for Hardy-Weinberg disequilibrium at a marker locus. Am J Hum Genet 63, 1531-1540; 1999.

Pfeiffer RM, Gail MH. Sample size calculations for population- and family-based case-control association studies on marker genotypes. Genet Epidemiol 25, 136$148 ; 2003$. 
Sasieni PD. From genotypes to genes: doubling the sample size. Biometrics 53, $1253-1261 ; 1997$.

Slager SL, Schaid DJ. Case-control studies of genetic markers: Power and sample size approximations for Armitage's test for trend. Hum Hered 52, 149-153; 2001.

Song K, Elston RC. A powerful method of combining measures of association and Hardy-Weinberg disequilibrium for fine-mapping in case-control studies. Statist Med 25, 105-126; 2006.

Todd, JA, Walker, NM, Cooper, JD, Smyth, DJ, Downes, K, Plagnol, V, Bailey, R, Nejentsev, S, Field, SF, Payne, F, et al. Robust associations of four new chromosome regions from genome-wide analyses of type 1 diabetes. Nat Genet, $39: 857-864 ; 2007$.

Vogel F, Motulsky AG. Human Genetics: Problems and Approaches. 2nd ed. Berlin, German: Springer-Verlag Heidelberg; 1986.

Zheng G, Joo J, Yang Y. Pearson's test, trend test, and MAX are all trend tests with different types of scores. Ann Hum Genet 73, 133-140; 2009.

Zheng G, Ng HKT. Genetic model selection in two-phase analysis for case-control association studies. Biostatistics 9, 391-399; 2008. 
Table 1: ORs for a given genetic model defined by GRRs with $\mathrm{GRR}_{2}=1.5$. The disease prevalence $\kappa=0.05,0.10,0.20 . x$ and $y$ are two indexes for the GRR and OR, respectively, which are the same as those in Equation 1 and Equation 2.

\begin{tabular}{|c|c|c|c|c|c|c|c|c|c|c|}
\hline \multirow[b]{2}{*}{$\kappa$} & \multirow[b]{2}{*}{ MAF } & \multicolumn{3}{|c|}{$\operatorname{REC}(x=0)$} & \multicolumn{3}{|c|}{$\operatorname{ADD}(x=1 / 2)$} & \multicolumn{3}{|c|}{$\operatorname{DOM}(x=1)$} \\
\hline & & $\mathrm{y}$ & OR1 & OR2 & $\mathrm{y}$ & OR1 & OR2 & $\mathrm{y}$ & OR1 & OR2 \\
\hline \multirow[t]{4}{*}{0.05} & 0.05 & 0 & 1 & 1.540 & 0.494 & 1.266 & 1.539 & 1 & 1.539 & 1.539 \\
\hline & 0.15 & 0 & 1 & 1.540 & 0.494 & 1.265 & 1.538 & 1 & 1.535 & 1.535 \\
\hline & 0.30 & 0 & 1 & 1.539 & 0.494 & 1.264 & 1.535 & 1 & 1.532 & 1.532 \\
\hline & 0.45 & 0 & 1 & 1.537 & 0.495 & 1.263 & 1.533 & 1 & 1.529 & 1.529 \\
\hline \multirow[t]{4}{*}{0.10} & 0.05 & 0 & 1 & 1.540 & 0.486 & 1.285 & 1.586 & 1 & 1.583 & 1.583 \\
\hline & 0.15 & 0 & 1 & 1.587 & 0.487 & 1.283 & 1.581 & 1 & 1.576 & 1.576 \\
\hline & 0.30 & 0 & 1 & 1.584 & 0.488 & 1.280 & 1.575 & 1 & 1.568 & 1.568 \\
\hline & 0.45 & 0 & 1 & 1.579 & 0.489 & 1.278 & 1.570 & 1 & 1.563 & 1.563 \\
\hline
\end{tabular}


Table 2: GRRs and ORs at the marker locus. True genetic model is defined at the disease locus using GRRs. Assume that disease and marker locus have the same MAF. Consider two LD coefficient: 1.0 and 0.9. $\kappa$ is the disease prevalence.

\begin{tabular}{|c|c|c|c|c|c|c|}
\hline \multirow[b]{2}{*}{$\kappa$} & \multirow[b]{2}{*}{ Model } & \multirow[b]{2}{*}{ MAF } & \multicolumn{2}{|c|}{$D^{\prime}=1.0$} & \multicolumn{2}{|c|}{$D^{\prime}=0.9$} \\
\hline & & & $\left(\mathrm{GRR}_{1}, \mathrm{GRR}_{2}\right)$ & $\left(\mathrm{OR}_{1}, \mathrm{OR}_{2}\right)$ & $\left(\mathrm{GRR}_{1}, \mathrm{GRR}_{2}\right)$ & $\left(\mathrm{OR}_{1}, \mathrm{OR}_{2}\right)$ \\
\hline \multirow[t]{12}{*}{0.05} & REC & 0.05 & $(1.00,1.50)$ & $(1.00,1.54)$ & $(1.00,1.41)$ & $(1.00,1.44)$ \\
\hline & & 0.15 & $(1.00,1.50)$ & $(1.00,1.54)$ & $(1.01,1.42)$ & $(1.01,1.45)$ \\
\hline & & 0.30 & $(1.00,1.50)$ & $(1.00,1.54)$ & $(1.01,1.43)$ & $(1.01,1.46)$ \\
\hline & & 0.45 & $(1.00,1.50)$ & $(1.00,1.54)$ & $(1.02,1.45)$ & $(1.02,1.48)$ \\
\hline & $\mathrm{ADD}$ & 0.05 & $(1.25,1.50)$ & $(1.27,1.54)$ & $(1.22,1.45)$ & $(1.24,1.48)$ \\
\hline & & 0.15 & $(1.25,1.50)$ & $(1.27,1.54)$ & $(1.22,1.45)$ & $(1.24,1.48)$ \\
\hline & & 0.30 & $(1.25,1.50)$ & $(1.26,1.53)$ & $(1.22,1.44)$ & $(1.23,1.47)$ \\
\hline & & 0.45 & $(1.25,1.50)$ & $(1.26,1.53)$ & $(1.22,1.44)$ & $(1.23,1.47)$ \\
\hline & DOM & 0.05 & $(1.50,1.50)$ & $(1.54,1.54)$ & $(1.45,1.49)$ & $(1.48,1.53)$ \\
\hline & & 0.15 & $(1.50,1.50)$ & $(1.54,1.54)$ & $(1.44,1.47)$ & $(1.47,1.51)$ \\
\hline & & 0.30 & $(1.50,1.50)$ & $(1.53,1.53)$ & $(1.42,1.46)$ & $(1.45,1.48)$ \\
\hline & & 0.45 & $(1.50,1.50)$ & $(1.53,1.53)$ & $(1.41,1.44)$ & $(1.44,1.46)$ \\
\hline \multirow[t]{12}{*}{0.10} & REC & 0.05 & $(1.00,1.50)$ & $(1.00,1.59)$ & $(1.00,1.41)$ & $(1.00,1.48)$ \\
\hline & & 0.15 & $(1.00,1.50)$ & $(1.00,1.59)$ & $(1.01,1.42)$ & $(1.01,1.49)$ \\
\hline & & 0.30 & $(1.00,1.50)$ & $(1.00,1.58)$ & $(1.01,1.43)$ & $(1.01,1.50)$ \\
\hline & & 0.45 & $(1.00,1.50)$ & $(1.00,1.58)$ & $(1.02,1.45)$ & $(1.02,1.51)$ \\
\hline & $\mathrm{ADD}$ & 0.05 & $(1.25,1.50)$ & $(1.28,1.59)$ & $(1.22,1.45)$ & $(1.25,1.52)$ \\
\hline & & 0.15 & $(1.25,1.50)$ & $(1.28,1.58)$ & $(1.22,1.45)$ & $(1.25,1.52)$ \\
\hline & & 0.30 & $(1.25,1.50)$ & $(1.28,1.58)$ & $(1.22,1.44)$ & $(1.25,1.51)$ \\
\hline & & 0.45 & $(1.25,1.50)$ & $(1.28,1.57)$ & $(1.22,1.44)$ & $(1.24,1.50)$ \\
\hline & DOM & 0.05 & $(1.50,1.50)$ & $(1.58,1.58)$ & $(1.45,1.49)$ & $(1.52,1.57)$ \\
\hline & & 0.15 & $(1.50,1.50)$ & $(1.58,1.58)$ & $(1.44,1.47)$ & $(1.50,1.55)$ \\
\hline & & 0.30 & $(1.50,1.50)$ & $(1.57,1.57)$ & $(1.42,1.45)$ & $(1.48,1.52)$ \\
\hline & & 0.45 & $(1.50,1.50)$ & $(1.56,1.56)$ & $(1.41,1.44)$ & $(1.46,1.49)$ \\
\hline
\end{tabular}


Table 3: The mean of $\hat{\theta}$ from 100,000 replicates simulated under a given genetic model with GRR $\lambda_{2}=1.5$. Denote the MAF as $p=0.05,0.15,0.30,0.45 . \kappa$ is the disease prevalence. The sample size is $n$ with equal number of cases and controls.

\begin{tabular}{|c|c|c|c|c|c|c|c|c|c|}
\hline \multirow[b]{2}{*}{ Model } & \multirow[b]{2}{*}{$n$} & \multicolumn{4}{|c|}{$\kappa=0.05$} & \multicolumn{4}{|c|}{$\kappa=0.10$} \\
\hline & & $p=0.05$ & 0.15 & 0.30 & 0.45 & $p=0.05$ & 0.15 & 0.30 & 0.45 \\
\hline \multirow[t]{5}{*}{ REC } & 200 & 1.092 & 0.462 & 0.064 & 0.089 & 1.084 & 0.471 & 0.036 & 0.041 \\
\hline & 500 & 1.787 & 0.191 & -0.062 & -0.080 & 1.770 & 0.157 & -0.022 & -0.173 \\
\hline & 1000 & 1.932 & -0.038 & -0.081 & -0.093 & 1.939 & 0.011 & -0.043 & -0.132 \\
\hline & 2000 & 1.464 & -0.032 & -0.042 & -0.063 & 1.442 & -0.088 & -0.037 & -0.066 \\
\hline & 5000 & 0.743 & 0.022 & -0.012 & -0.017 & 0.704 & -0.011 & -0.008 & -0.015 \\
\hline \multirow[t]{5}{*}{$\mathrm{ADD}$} & 200 & 1.000 & 0.482 & 0.390 & 0.503 & 1.023 & 0.521 & 0.408 & 0.503 \\
\hline & 500 & 1.692 & 0.321 & 0.580 & 0.524 & 1.693 & 0.355 & 0.583 & 0.493 \\
\hline & 1000 & 1.831 & 0.423 & 0.644 & 0.552 & 1.862 & 0.473 & 0.621 & 0.568 \\
\hline & 2000 & 1.480 & 0.753 & 0.576 & 0.564 & 1.475 & 0.695 & 0.622 & 0.557 \\
\hline & 5000 & 0.904 & 0.641 & 0.573 & 0.552 & 0.918 & 0.663 & 0.566 & 0.549 \\
\hline \multirow[t]{5}{*}{ DOM } & 200 & 0.981 & 0.415 & 0.678 & 0.789 & 0.963 & 0.447 & 0.726 & 0.877 \\
\hline & 500 & 1.585 & 0.294 & 1.021 & 1.019 & 1.613 & 0.535 & 1.034 & 1.082 \\
\hline & 1000 & 1.747 & 0.719 & 1.233 & 1.158 & 1.776 & 0.871 & 1.488 & 1.140 \\
\hline & 2000 & 1.421 & 1.092 & 1.077 & 1.076 & 1.448 & 1.040 & 1.160 & 1.084 \\
\hline & 5000 & 0.857 & 1.299 & 1.047 & 1.023 & 0.873 & 1.329 & 1.049 & 1.021 \\
\hline
\end{tabular}


Table 4: The model selection frequency (\%) of the genetic model using $Z_{c c}$ and $Z_{c}$ for a given genetic model with $\mathrm{GRR}_{2}=1.5$. Consider 1,000 cases and 1,000 controls. $\kappa$ is the disease prevalence.

\begin{tabular}{|c|c|c|c|c|c|c|c|c|}
\hline \multirow[b]{2}{*}{$\kappa$} & \multirow[b]{2}{*}{ MAF } & \multirow[b]{2}{*}{ Model } & \multicolumn{2}{|c|}{ under REC } & \multicolumn{2}{|c|}{ under ADD } & \multicolumn{2}{|c|}{ under DOM } \\
\hline & & & $Z_{c c}$ & $Z_{c}$ & $Z_{c c}$ & $Z_{c}$ & $Z_{c c}$ & $Z_{c}$ \\
\hline \multirow[t]{12}{*}{0.05} & 0.05 & REC & 15.83 & 21.62 & 4.41 & 6.21 & 0.81 & 1.27 \\
\hline & & $\mathrm{ADD}$ & 82.31 & 77.52 & 90.54 & 87.47 & 86.49 & 76.48 \\
\hline & & DOM & 1.86 & 0.86 & 5.05 & 6.32 & 12.70 & 22.25 \\
\hline & 0.15 & REC & 41.57 & 57.95 & 3.75 & 4.07 & 0.07 & 0.04 \\
\hline & & $\mathrm{ADD}$ & 58.27 & 42.02 & 89.62 & 87.01 & 54.78 & 32.46 \\
\hline & & $\mathrm{DOM}$ & 0.16 & 0.03 & 6.63 & 8.92 & 45.15 & 67.5 \\
\hline & 0.30 & REC & 70.93 & 89.76 & 3.22 & 3.26 & 0.01 & 0.01 \\
\hline & & $\mathrm{ADD}$ & 29.02 & 10.24 & 88.88 & 86.35 & 27.55 & 8.98 \\
\hline & & $\mathrm{DOM}$ & 0.05 & 0.00 & 7.90 & 10.39 & 72.44 & 91.01 \\
\hline & 0.45 & REC & 78.25 & 94.18 & 3.10 & 2.52 & 0.00 & 0.00 \\
\hline & & $\mathrm{ADD}$ & 21.75 & 5.82 & 88.76 & 87.12 & 23.18 & 5.62 \\
\hline & & $\mathrm{DOM}$ & 0.00 & 0.00 & 8.14 & 10.36 & 76.82 & 94.38 \\
\hline \multirow[t]{12}{*}{0.10} & 0.05 & REC & 16.31 & 20.93 & 4.33 & 6.71 & 0.85 & 1.39 \\
\hline & & $\mathrm{ADD}$ & 81.74 & 78.09 & 90.88 & 86.67 & 85.11 & 73.48 \\
\hline & & $\mathrm{DOM}$ & 1.95 & 0.98 & 4.79 & 6.62 & 14.04 & 25.13 \\
\hline & 0.15 & $\mathrm{REC}$ & 44.87 & 58.54 & 3.33 & 4.14 & 0.09 & 0.04 \\
\hline & & $\mathrm{ADD}$ & 54.98 & 41.42 & 90.24 & 87.53 & 51.41 & 32.31 \\
\hline & & $\mathrm{DOM}$ & 0.15 & 0.04 & 6.43 & 8.33 & 48.50 & 67.65 \\
\hline & 0.30 & REC & 76.18 & 89.64 & 3.22 & 2.76 & 0.00 & 0.00 \\
\hline & & $\mathrm{ADD}$ & 23.82 & 10.36 & 89.20 & 87.29 & 24.02 & 8.81 \\
\hline & & $\mathrm{DOM}$ & 0.00 & 0.00 & 7.58 & 9.95 & 75.98 & 91.19 \\
\hline & 0.45 & REC & 82.27 & 94.19 & 2.99 & 2.55 & 0.00 & 0.00 \\
\hline & & $\mathrm{ADD}$ & 17.73 & 5.81 & 89.14 & 87.31 & 19.81 & 5.25 \\
\hline & & $\mathrm{DOM}$ & 0.00 & 0.00 & 7.87 & 10.14 & 80.19 & 94.75 \\
\hline
\end{tabular}



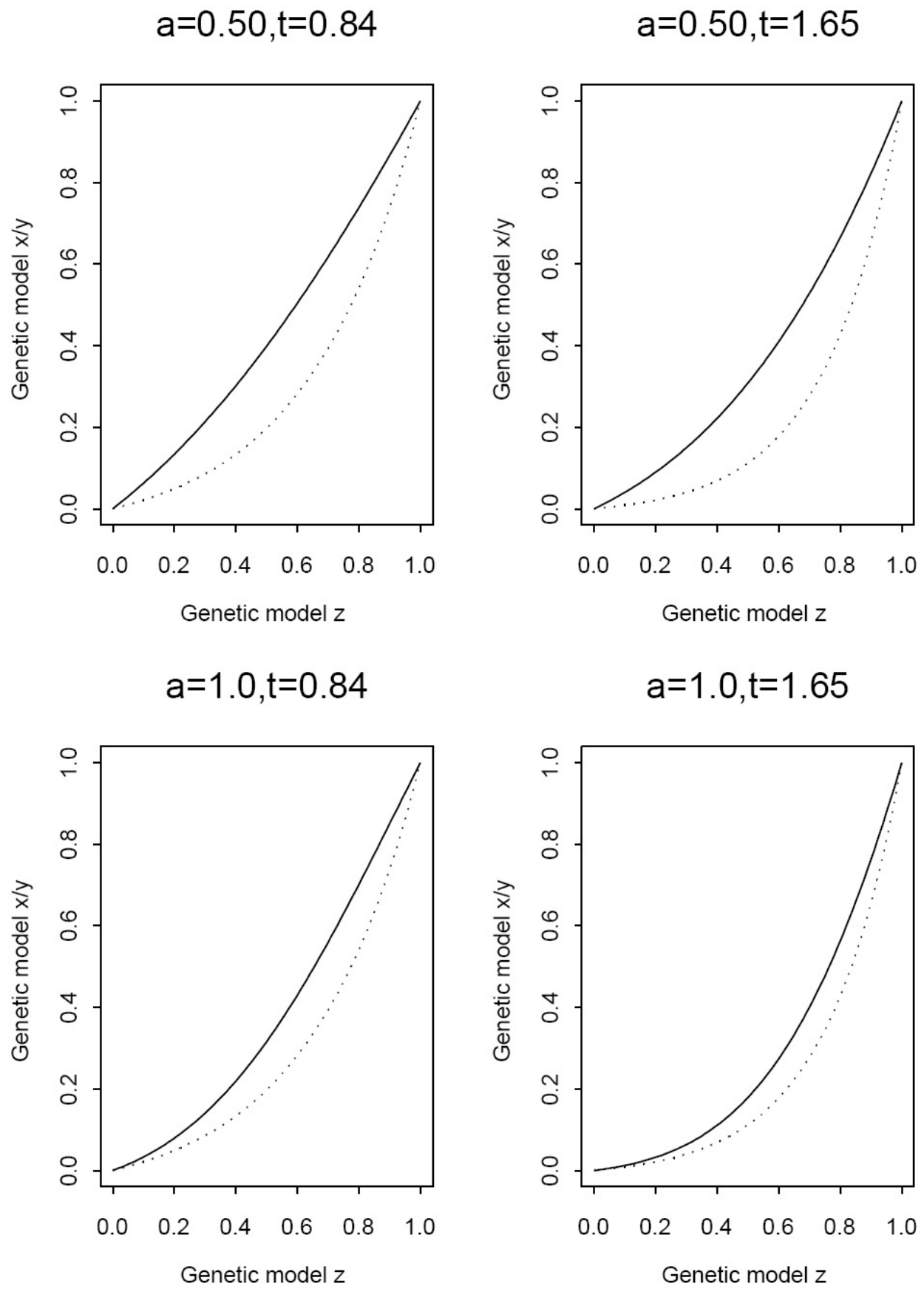

Figure 1: Plots of $x$ (solid line) and $y$ (dashed line) given $z$. The value $a$ and the normalized thresholds $t_{1}=-t$ and $t_{2}=t$ are given. Genetic model $\mathrm{x} / \mathrm{y}$ in the vertical axis means genetic model $\mathrm{x}$ or genetic model $\mathrm{y}$. genetic model $\mathrm{x}$ corresponds the solid line and y corresponds the dashed line. 